\title{
Reflecting on reflection: prospect theory, our behaviors, and our environment
}

\author{
Adam Oliver \\ Department of Social Policy, London School of Economics and Political Science, London, UK \\ E-mail: a.j.oliver@lse.ac.uk
}

(Received 13 September 2021; accepted 13 September 2021)

\begin{abstract}
In a previously published article, I reported some tests of prospect theory's reflection effect over outcomes defined by money and life years gained from treatment. Those results suggested qualified support for the reflection effect over money outcomes and strong support over longevity outcomes. This article reruns those tests while accounting for the intensity of individual risk attitudes, and, overall, show consistency with the reflection effect. However, I argue that these results do not necessarily offer support for the explanatory power of prospect theory. Rather, the results may be driven by evolved responses to circumstances that provoke perceptions of scarcity and abundance. Therefore, from an ecological perspective, behavioral patterns such as those that are consistent with the reflection effect, which, by extension, tend to be considered as erroneous or biased by most behavioral economists because they conflict with the postulates of rational choice theory, may not be unreasonable. Recognizing as such is important when considering how behavioral insights ought to inform public policy design and implementation.
\end{abstract}

Keywords: expected utility theory; prospect theory; reflection effect; risk intensity; risk sensitivity theory

JEL Classifications: B21; C12; C91

\section{Prospect theory's reflection effect}

Nobody working in behavioral public policy will be unfamiliar with prospect theory, with its component parts of probability weighting and, particularly, loss aversion having occupied prominent places in the discourse during the development of this relatively new multidisciplinary field. However, in the behavioral public policy literature (and indeed, in behavioral economics generally), little attention has been paid to what is, according to Tversky and Kahneman (1992), the most distinctive implication of their theory: specifically, the fourfold pattern of risk attitudes known as the reflection effect. Given prospect theory's general standing, this relative oversight is remiss. The purpose of this article is to give the reflection effect some much-needed exposure.

The combination of an individual's declining sensitivity to mounting gains and mounting losses and their subjective weighting of probability (such that they

(c) The Author(s), 2021. Published by Cambridge University Press. This is an Open Access article, distributed under the terms of the Creative Commons Attribution licence (http://creativecommons.org/licenses/by/4.0/), which permits unrestricted re-use, distribution and reproduction, provided the original article is properly cited. 
overweight low probabilities and underweight high probabilities), which are predicted by prospect theory, causes the reflection effect. The reflection effect is summarized in Table 1 - the effect offers opposing risk attitude predictions between gain and lossframed gambles when the probabilities of experiencing a gain/loss are high, and also when the probabilities are low.

Typically, a utility curve is constructed by plotting a respondent's certainty equivalent of a gamble against the expected value of that gamble, where the gamble takes the form of $\mathrm{p} \cdot \mathrm{x}+(1-\mathrm{p}) \cdot 0$, with $\mathrm{x}$ fixed, and repeating across the probability range. If this were done for $+\mathrm{x}$ and $-\mathrm{x}$, then we can construct a curve across the domains of both gains and losses. Figure 1 is a hypothetical depiction, where the black dotted line represents universal risk neutrality, the assumption that underpins standard rational choice theory. The red dotted curve is a representation that is consistent with the reflection effect: i.e., risk aversion over high probability gains and low probability losses, and risk seeking over low probability gains and high probability losses.

Controlled direct testing of the full reflection effect has been scarce and the results have been mixed. In a previously published article, I attempted to test reflection over both money and health-related outcomes - see Oliver (2018), in which much of the literature on reflection is reviewed. In those results, the evidence for the full reflection effect over money outcomes was also a little mixed, in that while the risk attitudes were strongly consistent with reflection when respondents were faced with high probability gambles, there was no general strong support for prospect theory reflection over small probability gambles. However, the results provided strong support for the full reflection effect over outcomes defined by longevity.

In my previous article, I tested only for the direction of each respondent's risk attitude in any question that they answered. As such, the response of someone who was, for example, only very marginally risk averse when faced with a particular gamble was given the same weight in testing the reflection effect as that given by a respondent who was highly risk seeking when presented with the same gamble. However, that the intensity of individual risk attitudes ought to count in tests of this kind cannot be dismissed lightly. Therefore, in part to further emphasize what the reflection effect predicts and in part to serve as some tests of robustness, I will hereby re-examine those results after taking into account the intensities of the respondents' risk attitudes.

\section{Reflecting on money}

The sample size was small and chosen for convenience - i.e., 60 postgraduate and university staff, 45 of whom were female (44 were age 18-30 years, 13 were $31-45$ years, one was 46-60 years and two were older than 60 years, and 44 had studied economics) - but no claim is made that the results are definitive. They are merely illustrative. Each respondent attended a face-to-face interview during which they were required to answer 30 questions. The questions were described as investment decisions, and their order was randomized across the respondents in a within-respondent design. To illustrate, two of the questions are replicated in Figure 2.

The respondents' near certainty equivalents were elicited for risky investment decisions that offered $90 \%, 70 \%, 50 \%, 30 \%$, and $10 \%$ chances of gaining and losing $£ 1$ million, $£ 10,000$, and $£ 100$. The respondents knew that the questions were financially 
Table 1. Prospect theory's reflection effect.

\begin{tabular}{lll}
\hline & Gains & Losses \\
\hline High Probability & Risk Aversion & Risk Seeking \\
\hline Low Probability & Risk Seeking & Risk Aversion \\
\hline
\end{tabular}

incentivised from the outset, but they were told that their payments would have to be scaled down from those indicated in the questions - the payments were normalized so that a maximum of $£ 1$ could be earned from each question, but the respondents were informed of the exact scaling only after they had answered the questions.

In a manner that mirrors that described for Figure 1, Figure 3 plots the respondents' median and mean certainty equivalents against the expected values of the gambles for each of the outcome magnitudes (i.e., £1million, $£ 10,000$, and $£ 100$ ). The black dotted lines imply universal risk neutrality, and the red and blue dotted curves respectively plot the respondents' median and mean certainty equivalents.

Particularly with respect to the means, the figure shows that the respondents were generally less accepting of risk for all three outcome magnitudes as probabilities declined in the domain of losses and as probabilities increased in the domain of gains, an observation that is consistent with prospect theory's reflection effect. Risk attitudes were more pronounced at the mean than at the median level, but for both measures risk neutrality was typically observed for low probability losses. It is, however, possible that the reflection effect's prediction of risk aversion in that area would have been more evident had percentage chances of losses lower than $10 \%$ been used in the tests. By far the strongest deviation from risk neutrality for all outcome magnitudes was risk seeking over high probability losses.

However, the medians plotted in the figure do not internalize the intensity of the risk attitudes. The means may better internalize intensity, which is perhaps why the mean curves are relatively more pronounced, but they are susceptible to distortions by outliers. Nonparametric tests of whether risk attitudes, after accounting for their intensity, differ significantly from risk neutrality, resolve these issues.

The intensity of each respondent's risk attitude in each question is calculated with:

$$
\frac{(\mathrm{CE}-\mathrm{EV})}{\mathrm{EV}}
$$

where $\mathrm{CE}$ is the respondent's certainty equivalent of a gamble and $\mathrm{EV}$ is the expected value of that gamble. If the individual is risk neutral, Equation $(1)=0$. Risk averse and risk seeking attitudes therefore produce a risk attitude intensity measure either side of zero. Tests of the respondents' attitudes toward risk were undertaken for all 30 questions, with the Wilcoxon matched pairs signed rank test used throughout. The results are summarized in Table 2.

In the table, the gambles that offered a chance of gaining $\mathfrak{E} 1$ million are summarized in the first five rows of the first column, and the respondents' collective risk attitude, accounting for the risk attitude intensity demonstrated by each respondent, is placed next to each gamble. The gambles that offered a chance of losing $£ 1$ million 


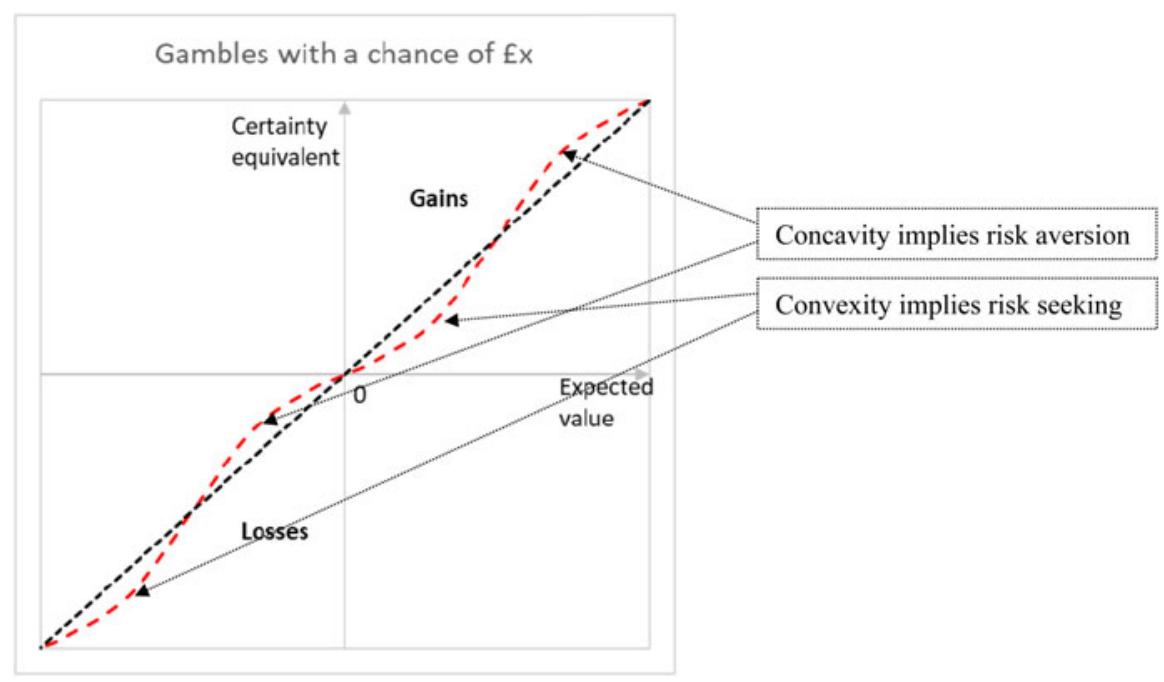

Figure 1. Diagrammatic Depiction of the Reflection Effect.

Question 1: You inherited an investment and there is a $90 \%$ chance that you could gain $£ 10,000$, with a $10 \%$ chance of gaining or losing nothing. You can, if you wish, sell this investment. What is the minimum amount of money for which you would be prepared to sell the investment?

Question 2: You inherited an investment and there is a $90 \%$ chance that you could lose $£ 10,000$, with a $10 \%$ chance of losing or gaining nothing. You can cancel this investment, but you will have to pay a penalty in order to do so. What is the maximum amount of money that you would be prepared to pay to cancel the investment?

Figure 2. The Questions Using Money Outcomes.

are summarized in the first five rows of the third column and the accompanying collective risk attitude is placed next to each of those. Similar presentations of the gambles that included outcome magnitudes of $£ 10,000$ and $£ 100$ are given in the middle and bottom thirds of the table, respectively.

On the whole, after taking into account the respondents' risk attitude intensities, the results in Table 2 are broadly consistent with those depicted in Figure 3. That is, for all three outcome magnitudes, the respondents generally became more averse to risk as probability increased in the domain of gains and as probability declined in the domain of losses, which, as noted earlier, is consistent with the predictions of the prospect theory reflection effect. The lowest probability gambles, in the domains of both gains and losses, sometimes reach only a point of risk neutrality in these tests when, at face value, one might expect risk seeking over gains and risk aversion over losses, but, 

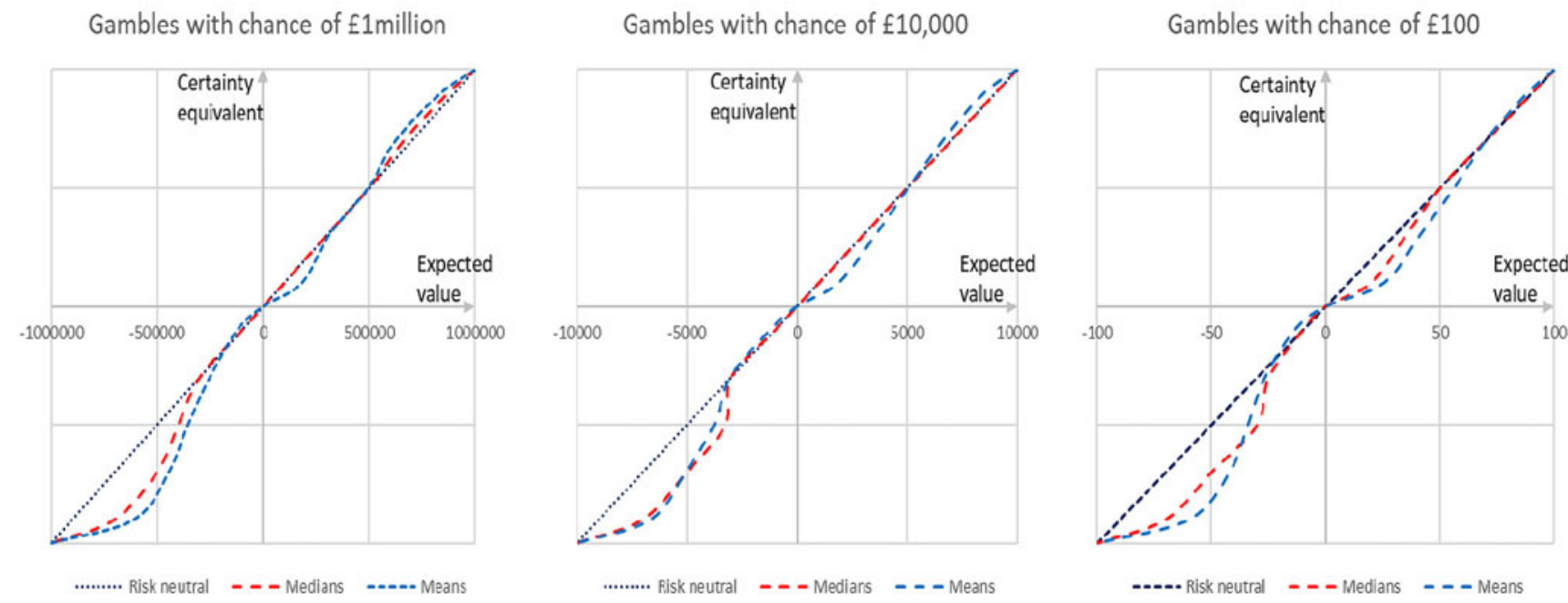

Figure 3. Diagrammatic Consistency with the Reflection Effect over Money Outcomes. 
Table 2. Results after accounting for intensity of risk attitude over money outcomes.

\begin{tabular}{|c|c|c|c|}
\hline Investment & Risk direction & Investment & Risk direction \\
\hline (£1million, 0.1) & Neutral & (-£1million, 0.1) & Neutral \\
\hline (£1million, 0.3) & Neutral & $(-£ 1 \text { million, } 0.3)^{\star}$ & Seeking \\
\hline (£1million, 0.5) & Neutral & $(-£ 1 \text { million, } 0.5)^{\star}$ & Seeking \\
\hline$(£ 1 \text { million, } 0.7)^{\star}$ & Aversion & $(-£ 1 \text { million, } 0.7)^{\star}$ & Seeking \\
\hline (£1million, 0.9)* & Aversion & $(-£ 1 \text { million, } 0.9)^{\star}$ & Seeking \\
\hline$(£ 10,000,0.1)^{*}$ & Seeking & $(-£ 10,000,0.1)$ & Neutral \\
\hline$(£ 10,000,0.3)^{\star}$ & Seeking & $(-£ 10,000,0.3)$ & Neutral \\
\hline$(£ 10,000,0.5)$ & Neutral & $(-£ 10,000,0.5)^{\star}$ & Seeking \\
\hline$(£ 10,000,0.7)^{\star \star}$ & Aversion & $(-£ 10,000,0.7)^{\star}$ & Seeking \\
\hline$(£ 10,000,0.9)^{\star}$ & Aversion & $(-£ 10,000,0.9)^{\star}$ & Seeking \\
\hline$(£ 100,0.1)^{\star}$ & Seeking & $(-£ 100,0.1)^{\star}$ & Aversion \\
\hline$(£ 100,0.3)^{\star}$ & Seeking & $(-£ 100,0.3)^{\star \star}$ & Seeking \\
\hline$(£ 100,0.5)^{\star}$ & Seeking & $(-£ 100,0.5)^{\star}$ & Seeking \\
\hline$(£ 100,0.7)$ & Neutral & $(-£ 100,0.7)^{\star}$ & Seeking \\
\hline$(£ 100,0.9)^{\star \star}$ & Aversion & $(-£ 100,0.9)^{\star}$ & Seeking \\
\hline
\end{tabular}

${ }^{\star} 1 \%$ significance.

${ }^{*} 5 \%$ significance.

again, this may be because the lowest probabilities used in these tests were not always sufficiently low enough for the respondents to depart significantly and systematically from the position of risk neutrality. As also observed in Figure 3, the most intense and consistent risk attitude was that of risk seeking in the face of high - and not always high - probability losses, an attitude observed universally at a $1 \%$ level of significance.

My previously published article was I think the first to report tests of fourfold reflection over health-related longevity, with the full effect holding up very well in those. However, we (or rather, I) ought to examine whether those conclusions persist after also accounting for the intensity of the respondents' risk attitudes.

\section{Reflecting on health}

A similar, but different, sample of respondents answered the health-related questions - i.e., 60 postgraduates and university staff, 45 of whom were female (47 were age 1830 years and 13 were $31-45$ years, and 44 had studied economics). Each respondent attended a face-to-face interview during which they answered 30 hypothetical health care-related questions. The order of the questions was randomized across the respondents in a within-respondent design, and two of the questions are replicated in Figure 4 .

The respondents near certainty equivalents were elicited for risky healthcare treatments offering $90 \%, 70 \%, 50 \%, 30 \%$, and $10 \%$ chances of gains and losses of 480 
months, 180 months, and 36 months of life. The respondents were paid a flat fee of $£ 10$ for their participation and were asked to assume that additional longevity would be experienced in full health. Months rather than years of life were chosen in the hope that this would cause the respondents to fine-tune their answers. Figure 5 plots the respondents' median and mean certainty equivalents against the expected values of the healthcare treatments for each of the outcome magnitudes.

If one focusses upon the medians, the figure suggests that the respondents did not stray too far from risk neutrality, except when faced with high probability losses. The means, as with the study that used money outcomes, offer a more pronounced impression of a set of risk attitudes that are evidently consistent with the predictions of the prospect theory reflection effect. As was also the case with the tests using money outcomes, if one considers the medians and means together, the standout departure from risk neutrality is risk seeking over large probability losses.

Table 3 summarizes the results of the tests of the reflection effect over longevity outcomes after accounting for the intensity of the respondents' risk attitudes. In all cases where attitudes depart from risk neutrality they do so at a high level of statistical significance, and the risk attitude patterns as probability increases is consistent with the predictions of the prospect theory reflection effect in the domain of both gains (i.e., risk seeking to risk aversion) and losses (i.e., risk aversion to risk seeking) across all three outcome magnitudes. Therefore, at face value, these tests over longevity offer strong support for prospect theory. But does prospect theory really explain these results?

\section{Ecological influences}

Placing to one side the many methodological objections that people may have to my experiments (I apologize that I could not actually offer people the chance to win or lose $£ 1$ million, and that I could not place people in life-limiting conditions, and those who are unconvinced by any study that is not fully incentivised are free, or course, to ignore this article), the results presented above are generally consistent with the predictions of prospect theory. However, although the predictive validity of prospect theory with respect to these particular results is quite high, its performance as an explanatory theory depends on whether people really do assess risky options by somewhat mechanistically weighting the subjective value of their outcomes with the associated subjective probabilities. Might it instead be the case that people's responses in these tests were influenced heavily by deeply ingrained survival instincts that evolved in relation to perceptions of whether available (food) resources are scarce or abundant?

The hypothesis of risk aversion in the face of abundance and risk seeking in the face of extreme scarcity to facilitate survival is postulated by risk sensitivity theory, developed by behavioral ecologists to explain rational food acquisition decisionmaking by foraging animals in an uncertain environment. Mishra (2014), for example, notes that risk aversion will switch to risk seeking when a nutritional need has to be fulfilled and which cannot be met with a low risk option; thus, rather than outcomes maximization over repeated tasks, the evolved emphasis - which may still today be rational in many circumstances - is on attempting to meet one's basic needs in the moment. 
Question 1: You have an illness which will kill you within the next few days unless you take a treatment. Two treatments are available to you. If you take treatment $\mathrm{A}$, there is a $90 \%$ chance that you will live for 36 more months and then die, and a $10 \%$ chance that the treatment will not work and you will die within the next few days. Alternatively, treatment B offers a number of months of life for certain, but we do not know how many months that is.

What is the minimum number of months you would require from treatment $\mathrm{B}$ in order for you to just prefer to take B over A?

Question 2: You are someone who would normally expect 36 more months of life. Unfortunately, your situation is not normal, and you need to take a health care treatment. Without treatment, you are certain to lose all 36 months. With treatment A, you still face a $90 \%$ chance of losing the 36 months, but have a $10 \%$ chance of maintaining your normal life expectancy. Your doctor has offered you alternative treatment B where you are certain to lose a number of months from your normal life expectancy.

How many months of your normal life expectancy are you willing to forgo in order to be tempted to take B over A?

Figure 4. The Questions Using Longevity Outcomes.

If probability serves as a proxy for prior (and thus expected) frequency of success in the respondents' psychologies as they process the risky decisions that they face, then the top and bottom left quadrants of Table 1 - high and low probabilities of a gain - may respectively provoke perceptions of abundance and scarcity; perceptions that are central to the explanatory power of risk sensitivity theory. With abundance, people may be quite satisfied with an implicitly risk averse strategy - accepting a guaranteed amount that is less than the expected value of the gamble suffices. However, with scarcity the expected value of a gamble that offers a small chance of a gain is insufficient to meet one's needs or aspirations. It is only by accepting the gamble that one has any chance at all of seeing those needs or aspirations met. If people implicitly also perceive the bottom and top right quadrants of Table 1 high and low probabilities of a loss - as situations that suggest scarcity and abundance (i.e. if a loss is interpreted as a failure to meet one's needs or aspirations), risk sensitivity theory offers an explanation for the patterns of risk attitude that are consistent with the full reflection effect.

It may be the case that the size of the outcome rather than the probability of success is sometimes the dominant factor in influencing perceptions of scarcity and abundance. For example, if there is a good chance of experiencing what might be considered a very small gain, the perception might be one of scarcity rather than abundance, which may thus provoke actions and behaviors that are consistent with risk seeking rather than risk aversion. Thus, it is possible that the consideration of either extremely small or extremely large outcomes might lead to ecologically rational choices that are inconsistent with the predictions of prospect theory. For instance, although the expected value of a large probability of a very small outcome might strike most people as neither here nor there, thus causing them to opt for the risk, 
Treatment with chance of 480 months

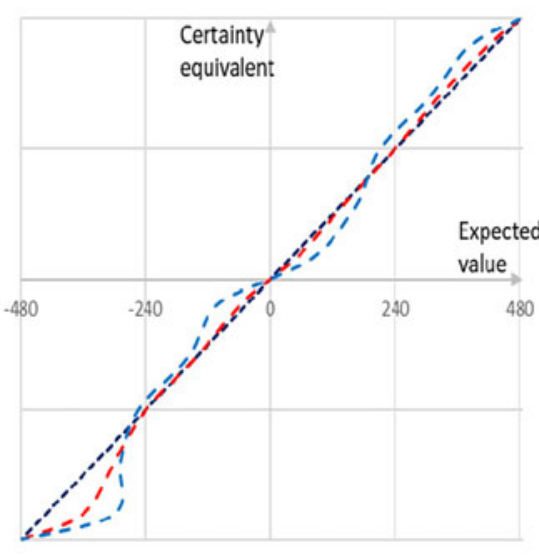

-----Risk neutral - - - Medians $\quad---$ Means
Treatment with chance of 180 months

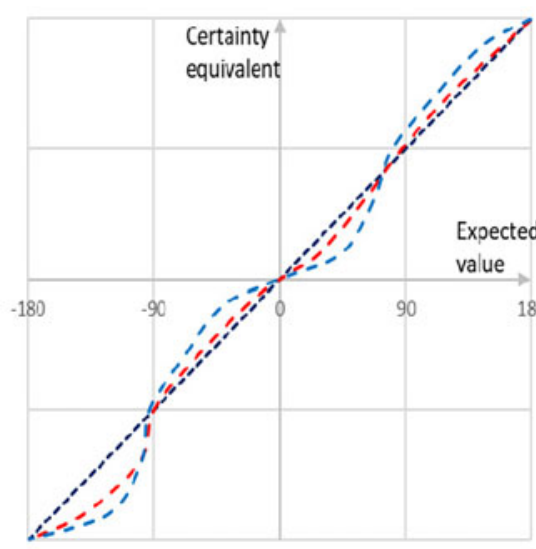

-.-.- Fisk neutral _- - Mediars - - - Means
Treatment with chance of 36 months

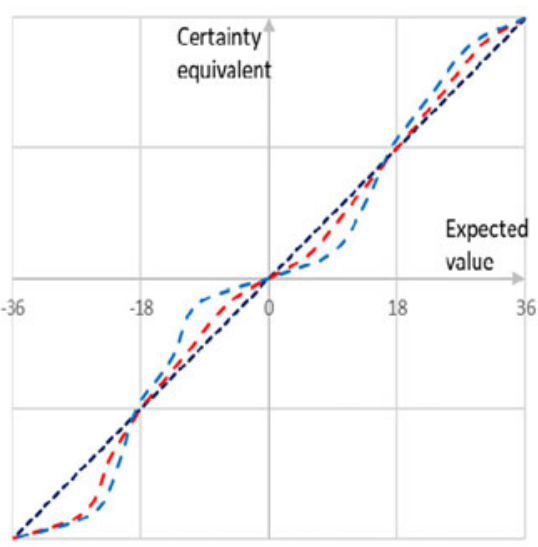

-.-.- Risk neutral _ - - Medians - - - Means 
Table 3. Results after accounting for intensity of risk attitude over longevity outcomes.

\begin{tabular}{|c|c|c|c|}
\hline Treatment & Risk direction & Treatment & Risk direction \\
\hline (480 months, 0.1$)^{*}$ & Seeking & $(-480 \text { months, } 0.1)^{\star}$ & Aversion \\
\hline (480 months, 0.3 ) & Neutral & ( -480 months, 0.3 ) & Neutral \\
\hline$(480 \text { months, } 0.5)^{*}$ & Aversion & (-480 months, 0.5$)$ & Neutral \\
\hline (480 months, 0.7$)^{\star}$ & Aversion & $(-480 \text { months, } 0.7)^{\star}$ & Seeking \\
\hline (480 months, 0.9$)^{*}$ & Aversion & $(-480 \text { months, } 0.9)^{\star}$ & Seeking \\
\hline$(180 \text { months, } 0.1)^{*}$ & Seeking & $(-180 \text { months, } 0.1)^{\star}$ & Aversion \\
\hline$(180 \text { months, } 0.3)^{*}$ & Seeking & $(-180 \text { months, } 0.3)^{*}$ & Aversion \\
\hline$(180 \text { months, } 0.5)^{*}$ & Aversion & (-180 months, 0.5$)$ & Neutral \\
\hline$(180 \text { months, } 0.7)^{\star}$ & Aversion & $(-180 \text { months, } 0.7)^{\star}$ & Seeking \\
\hline$(180 \text { months, } 0.9)^{*}$ & Aversion & $(-180 \text { months, } 0.9)^{\star}$ & Seeking \\
\hline$(36 \text { months, } 0.1)^{\star}$ & Seeking & $(-36 \text { months, } 0.1)^{*}$ & Aversion \\
\hline (36 months, 0.3$)^{\star}$ & Seeking & $(-36 \text { months, } 0.3)^{\star}$ & Aversion \\
\hline (36 months, 0.5) & Neutral & (-36 months, 0.5$)$ & Neutral \\
\hline$(36 \text { months, } 0.7)^{\star}$ & Aversion & $(-36 \text { months, } 0.7)^{*}$ & Seeking \\
\hline$(36 \text { months, } 0.9)^{*}$ & Aversion & $(-36 \text { months, } 0.9)^{*}$ & Seeking \\
\hline
\end{tabular}

${ }^{\star} 1 \%$ significance.

the expected value of a small probability of an extremely large outcome may suffice for most people, implying risk aversion. These behavioral tendencies might invoke a different type of reflection effect than that predicted by prospect theory, and yet can still be explained by risk sensitivity theory.

Relatedly, risk sensitivity theory can also explain Markowitz's (1952) hypothesis pertaining to risk attitudes. Markowitz proposed that at, say, a $10 \%$ chance of winning or losing, people will be risk averse over gambles that offer large gains or small losses (e.g., respectively, preferring $£ 10,000,000$ for sure over a $10 \%$ chance of $£ 100,000,000$, and preferring losing $£ 10$ p for sure over a $10 \%$ chance of losing $£ 1$ ), and risk seeking over gambles that offer small gains and large losses (e.g., respectively, preferring a $10 \%$ chance of $£ 1$ over $10 \mathrm{p}$ for sure, and preferring a $10 \%$ chance of losing $£ 100,000,000$ over losing $£ 10,000,000$ for sure). Gambles that present particularly large gains or small losses might be interpreted as scenarios of abundance, while those that present particularly small gains and large losses are perhaps more likely to be perceived as scenarios of scarcity (depending on where one's reference point lies). Risk sensitivity theory thus suggests that if one takes a broad ecological perspective, seemingly inconsistent predictions made in the behavioral economics literature, such as those offered by the Markowitz and prospect theory hypotheses, may be explained under a single framework.

The results presented in this article in support of the prospect theory reflection effect may therefore be an artifact of the experiment's design (e.g., the size of the probabilities and outcomes used), and might not actually be explained by prospect 
theory. A more general ecological explanation encompasses both these and other results. If the reflection effect, and indeed the whole gamut of predictions that are made within the field of behavioral economics, are usefully to extend their increasing and already substantial input into public policy design, it would make sense to try to reinforce those predictions with genuine explanatory depth.

For instance, a risk seeking tendency, predicted by prospect theory and often observed in human decision-making, has been attributed to errors and biases in human decision-making, and these tendencies can certainly be manipulated to impose harms upon people, as the gambling industry - and, in particular, the rise of online gambling - attests. But if one tries to understand how a person's circumstances and environment motivates him or her to behave, act, and decide in particular ways, one might appreciate that their inconsistencies with standard notions of rational choice are not necessarily irrational in a more inclusive sense of the term. A policy maker might then realize that some people might rationally choose to gamble, for example, because they believe that this is the only possible way for them to escape a particular financial predicament. If their situation is already dire in the absence of gambling, then attempting to regulate their gambling behaviors in some way without other supporting mechanisms may do little to assist them.

To sum up, behavioral economists and relevant psychologists have over the past several decades done a good job at identifying systematic behavioral patterns, but their attempts at explaining their findings have generally been a little perfunctory. As a discipline, they (or rather, we) need to escape from the overriding belief that all that conflicts with rational choice theory is erroneous and biased, or, in other words, that the maximization of utility (or welfare or happiness) is necessarily the appropriate normative standard to pursue. The findings uncovered by behavioral economists may be perfectly reasonable responses given the circumstances and the environment that people find themselves in, and recognizing as such ought to influence how these findings should be used, or not used, to inform public policy.

Conflict of interest. The author declares no conflicts of interest.

\section{References}

Markowitz, H. (1952), 'The utility of wealth', Journal of Political Economy, 60(2): 151-158.

Mishra, S. (2014), 'Decision-making under risk: Integrating perspectives from biology, economics and psychology', Personality and Social Psychology Review, 18(3): 280-307.

Oliver, A. (2018), 'Your money and your life: Risk attitudes over gains and losses', Journal of Risk and Uncertainty, 57(1): 29-50.

Tversky, A. and D. Kahneman (1992), 'Advances in prospect theory: Cumulative representation of uncertainty', Journal of Risk and Uncertainty, 5(4): 297-323.

Cite this article: Oliver A (2021). Reflecting on reflection: prospect theory, our behaviors, and our environment. Behavioural Public Policy 1-11. https://doi.org/10.1017/bpp.2021.31 